\title{
Using Demographic Models to Determine Intrinsic Rate of Increase and Sustainable Fishing for Elasmobranchs: Pitfalls, Advances, and Applications
}

\author{
Todd Gedamke, John M. Hoenig, ${ }^{*}$ John A. Musick, and William D. DuPaul \\ Virginia Institute of Marine Science, College of William and Mary, \\ Post Office Box 1346, Route 1208 Greate Road, Gloucester Point, Virginia 23062, USA
}

\author{
SAmuel H. Gruber \\ Rosensteil School of Marine and Atmospheric Sciences, University of Miami, \\ 4600 Rickenbacker Causeway, Miami, Florida 33149, USA
}

\begin{abstract}
Leslie matrices and life tables are demographic models commonly used to evaluate the ability of specific elasmobranch life history strategies to sustain given levels and patterns of fishing pressure. These models are generally density independent and provide an instantaneous rate of population growth for a specified set of life history traits that correspond to a specific population size. Many investigators are using these models to compute rates of population growth that they claim are estimates of the maximum population growth rate $\left(r_{\text {intrinsic }}\right)$; they then use these estimates to compute purported estimates of maximum sustainable fishing mortality. However, neither a Leslie matrix nor a life table can be used to estimate $r_{\text {intrinsic }}$ without additional information, except in the special case where a severely depleted population is modeled. Only in a severely depleted population will competition for resources be at a minimum and both density-dependent compensation and the rate of population growth be at a maximum (i.e., at $r_{\text {intrinsic }}$ ). The fundamental problem is to determine the life history parameters that would occur if the population were extremely depleted because extensive observations on extremely depleted populations are rare. In the absence of such data, $r_{\text {intrinsic }}$ can only be estimated from these types of density-independent models by extrapolating observed population growth rates toward zero population size. We illustrate the problems in, and describe methods for, estimating $r_{\text {intrinsic }}$ and present information on two species of elasmobranch: barndoor skate Dipturus laevis and lemon shark Negaprion brevirostris.
\end{abstract}

Classic demographic analysis, based on a life table or Leslie matrix, provides an estimate of the exponential (or, more properly, geometric) rate of population growth or decline based on a fixed set of life history parameters. Alternatively, the model can be thought of as providing the current (short-term) rate of population change under current conditions. For the elasmobranchs, where many stocks have been severely depleted, the question is to what extent these populations can withstand fishing pressure. Recently, this question has been approached by attempting to use demographic models to determine the intrinsic or maximum rate of population increase $\left(r_{\text {intrinsic }}\right)$ and therefore the maximum sustainable fishing pressure. However, fundamental errors in the interpretation of the models are common.

Problems in the use of a basic demographic analysis for estimating $r_{\text {intrinsic }}$ arise from the density-independent nature of its structure and the use of static life history parameter inputs. In reality, at least some life

\footnotetext{
* Corresponding author: hoenig@vims.edu
}

Received September 12, 2005; accepted January 4, 2007 Published online April 26, 2007 history traits must be pliable and able to respond to changes in population size. This forms the basic logic behind density-dependent compensation, which explains why populations rarely go extinct and cannot grow beyond the bounds fixed by limiting factors (such as food resources or space) for extended periods (i.e., there is a carrying capacity of the environment).

Throughout most of this paper, we will assume for simplification of exposition that all compensatory response occurs in the survival in the first year of life $\left(S_{0}\right)$ rather than in maturity, fecundity, or survival after the first year. This may be particularly justifiable in the case of viviparous elasmobranchs, as it is unlikely that litter size or mating frequency could change appreciably, and $S_{0}$ appears to be related to population size in at least one of the species on which we focus, the lemon shark Negaprion brevirostris (Gruber et al. 2001). When evidence exists that compensation occurs in other parameters, this is easily incorporated into the models. In our analysis of the lemon shark (see Application to Elasmobranchs below), for example, we had evidence to support extending the compensatory response into the survival of age-1 animals. For oviparous elasmobranchs with an egg stage duration 
approaching a year, the mechanism for compensation may be more complex, involving fecundity (eggs/year), egg survival $\left(S_{\text {egg }}\right)$, and $S_{0}$. We deal with this added complexity in our analysis of the barndoor skate Dipturus laevis (see Advances in Methodology and Application to Elasmobranchs).

Direct evidence for density-dependent relationships is rarely available. In fact, even estimating the survival of the youngest animals is extremely difficult regardless of population size. Therefore, these models can be used in reverse, allowing first-year survival $\left(S_{0}\right)$ to be calculated assuming a schedule of reproductive output and survival (excluding $S_{0}$ ) and a known rate of population growth (e.g., a $10 \%$ rate of increase might be observed when a stock is released from fishing pressure or zero growth might be observed or hypothesized when the population is at equilibrium; Vaughan and Saila 1976). Hoenig and Gruber (1990) expanded on this approach by estimating $S_{0}$ for a virgin population of lemon sharks assumed to be at equilibrium. They assumed that changes in $S_{0}$ are the principal mechanism for density-dependent compensation, and for a series of increasing values of fishing mortality, calculated the value of $S_{0}$ that would result in equilibrium. Since it is unlikely that first-year survival can rise to the level for unfished adults, the value of fishing mortality that would require the first-year survival to rise above that of an adult in an unfished population was interpreted as an upper limit to sustainable fishing mortality. Similarly, an upper limit to the intrinsic rate of population growth $\left(r_{\text {intrinsic }}\right)$ is estimated by removing fishing pressure from this model. However, there is no guarantee that these upper limit estimates can be achieved.

A number of studies have followed this upper-limit approach (Sminkey and Musick 1996; Casey and Myers 1998; Mollet and Cailliet 2002). However, Cortés' (2004) summary of studies using demographic analysis for elasmobranchs reveals that many investigators either (1) purported to calculate an actual value for $r_{\text {intrinsic }}$ (or, equivalently, to calculate maximum sustainable fishing pressure) rather than an upper bound or (2) tried to rank species by the amount of fishing mortality they can withstand. The logic behind these attempts is generally unclear and is either flawed or based on unstated and unrealistic assumptions.

In this paper we first review the logic of simple demographic analysis using the Leslie matrix (the same logic holds for life tables), show basic relationships, and discuss what can and cannot be inferred. We then discuss how additional information can be utilized to make additional inferences. We derive methods for estimating $r_{\text {intrinsic }}$ and apply them to two elasmobranch species, barndoor skate and lemon shark.

\section{Basics of Population Dynamics and Demographic Analysis}

Demographic analysis simply tracks the change over time in the number of animals at different ages or stages given a schedule of age- or stage-specific reproductive output and mortality (Gotelli 1998; Caswell 2001). Models can be constructed assuming continuous or annual reproduction and, in the latter case, assuming that abundances pertain to the period just before or just after breeding occurs.

Suppose we have the following information, which would be required for an age-based demographic analysis of a viviparous species: age at maturity $\left(a_{\text {mat }}\right)$ $=3$ years, longevity $\left(a_{\max }\right)=6$ years, survivorship at each age $\left(S_{0}, S_{1}, S_{2}, \ldots, S_{5}\right)$, and the production of females per female $\left(f_{1}, f_{2}, f_{3}, \ldots, f_{6}\right)$, which is a function of the percentage reproducing in each ageclass, the frequency of births, the sex ratio, and litter size. We assume that the numbers are tallied before reproduction takes place (a prebreeding census). We can then calculate from the number of females at each age $\left(n_{1, t}, n_{2, t}, \ldots, n_{6, t}\right)$ the number there will be in the following year $\left(n_{1, t+1}, n_{2, t+1}, \ldots, n_{6, t+1}\right)$, where $n_{i j}$ is the number of animals of age $i$ at the start of year $j$. The number of age- 0 females produced will be

$$
n_{1, t+1}=S_{0} \sum_{i=1}^{6} n_{i, t} f_{i} .
$$

The number of females at all other ages is given by

$$
n_{i+1, t+1}=n_{i, t} S_{i}
$$

Often, the age-specific fecundities can be modeled, at least approximately, as the product of the age-specific proportion of mature adults $\left(P_{i}\right)$ and a constant fecundity per mature adult $(f)$, such that $f_{i}=P_{i} f$. This assumes that the fecundity of mature animals does not change with age.

These basic relationships are fundamental to any demographic analysis, including life tables, matrix analysis, and Euler-Lotka approaches. In a Leslie matrix analysis, the life history information for our example is organized in a projection matrix $\mathbf{A}$ as follows:

$$
\mathbf{A}=\left[\begin{array}{cccccc}
0 & 0 & S_{0} f_{3} & S_{0} f_{4} & S_{0} f_{5} & S_{0} f_{6} \\
S_{1} & 0 & 0 & 0 & 0 & 0 \\
0 & S_{2} & 0 & 0 & 0 & 0 \\
0 & 0 & S_{3} & 0 & 0 & 0 \\
0 & 0 & 0 & S_{4} & 0 & 0 \\
0 & 0 & 0 & 0 & S_{5} & 0
\end{array}\right]
$$

For an oviparous species with an egg stage lasting approximately 1 year, the matrix would have an 
additional row and column to accommodate the egg stage. The first row of the matrix would have entries representing the product of fecundity (female eggs/ year) and egg survival. The second row would have $S_{0}$ in the first column and zeros elsewhere, and each subsequent row would have a single survival rate corresponding to the next age-class.

The number of females at each age at time $t$ is denoted by

$$
\mathbf{N}_{t}=\left[n_{1, t}, n_{2, t}, n_{3, t}, n_{4, t}, n_{5, t}, n_{6, t}\right]^{\mathrm{T}} .
$$

The female population at time $t+1$ is then given by

$$
\mathbf{N}_{t+1}=\mathbf{A} \mathbf{N}_{t} \text {. }
$$

The predicted rate of population increase $\left(r_{\text {predicted }}\right)$ is defined here to be the instantaneous rate of growth of the population given the parameters used in the projection matrix and a stable age distribution. It is directly related to the largest eigenvalue $(\lambda)$ of the matrix $\mathbf{A}$ as $r_{\text {predicted }}=\log _{e}(\lambda)$ (Vaughan and Saila 1976). In a follow-up study, Vaughan (1977) derived a computational method to calculate $r_{\text {predicted }}$ that is often used in practice.

In this type of demographic model, $r_{\text {predicted }}$ represents a snapshot of the population growth rate based on a fixed set of life history parameters and a given schedule of fishing mortality. In reality, populations are not governed by a fixed set of life history parameters but by the dynamic relationship of these parameters to stock size. The basic logistic model of population growth has been used extensively in both fisheries and ecological research and is the simplest to include density-dependent compensation through a linear relationship of the per capita population growth rate to population size. Throughout this paper, the logistic model will simply serve to illustrate the importance of including stock size in the design and interpretation of a demographic model. In reality, the relationship may be curvilinear (see, for example, Sibley et al. 2005), but use of a nonlinear model requires more data, which will often not be available.

The logistic model (and density-dependent models in general) states that under virgin conditions a population will reach an equilibrium state around a carrying capacity ( $K$; Figure 1a). The number of births will equal the number of deaths and the population growth rate will equal zero until some force, such as fishing pressure, reduces the population size. At lower numbers, more resources are available to each individual and survival, particularly that of first-year individuals, increases. If the population is released from the fishing pressure, a recovery begins at a rate that is conditional on the population size relative to the virgin stock size. The logistic model states that the instantaneous per capita rate of growth will be greatest in an uncrowded condition. As the population recovers and resources become increasingly limiting, population growth slows and eventually approaches zero (i.e., births equal deaths). In reality, virgin populations may not have a zero growth rate at any particular point in time. However, we stress that the expected or longterm average growth rate must be zero.

We use the following definitions of instantaneous population growth rates in this paper:

$r_{\text {intrinsic }}=$ the maximum per capita population growth rate (this can only occur in the absence of fishing at the lowest population size, when density-dependent compensation is at a maximum); a stable age distribution is assumed;

$r_{\text {conditional }}=$ the per capita rate of population growth when there is no fishing mortality, given a schedule of survival and reproduction (conditional on population size and the resulting density-dependent compensation) and given a stable age distribution. In a logistic model, $r_{\text {conditional }}=r_{\text {intrinsic }} \cdot(1$ $-N / K)$, where $N$ is the total population size and $K$ is the carrying capacity;

$r_{\text {predicted }}=$ the predicted rate of population growth per capita when all other parameters are known (assuming a stable age distribution and a given population size); $r_{\text {predicted }}$ equals $r_{\text {conditional }}$ minus any effects of fishing mortality, and

$r_{\text {achieved }}=$ the per capita rate of population growth; no assumption of a stable age distribution is made.

It is important to note that the rate of population growth achieved in the field $\left(r_{\text {achieved }}\right)$ is expected to equal $r_{\text {predicted }}$ when a stable age distribution is present in the population (Figure 2).

The principle that population growth rate is linked to population abundance is critical to the design and interpretation of any elasmobranch demographic analysis (Vaughan 1977; Hoenig and Gruber 1990). Consider a hypothetical elasmobranch population under three different levels of exploitation: virgin, moderately fished, and depleted (Figure 1b). Assume that the fishing history has been stable for long enough that by time $a$ any density-dependent compensation that could occur has occurred and the populations are at equilibrium. The three populations will have realized different levels of density-dependent compensation to remain at equilibrium at different population sizes and 
a.

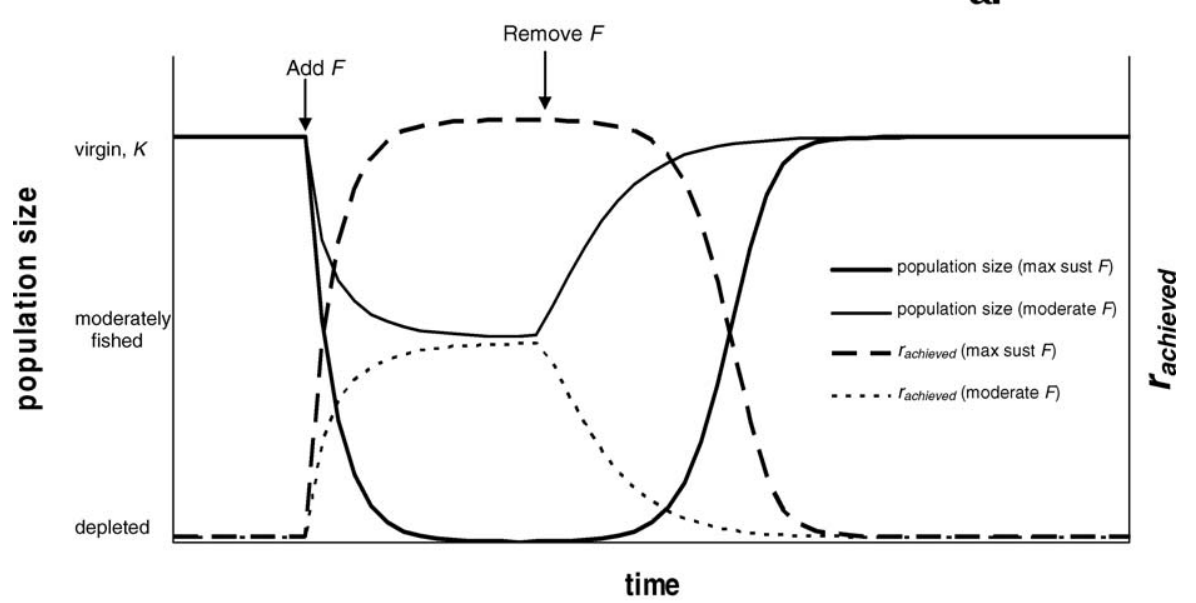

b.

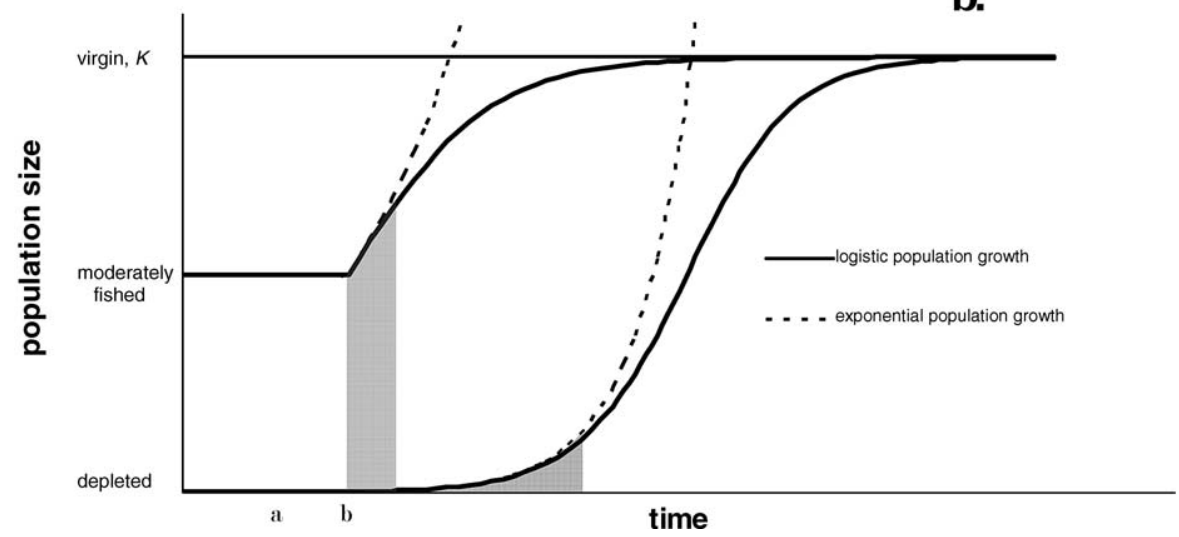

FIGURE 1.-Panel (a) shows the population dynamics and density-dependent responses in the per capita rate of population growth $\left(r_{\text {achieved }}\right)$ to the addition and removal of two levels of constant fishing mortality $(F)$ according to the logistic model of population growth (max sust $F=$ maximum sustainable fishing mortality). Panel (b) shows the steady state (time $a$ ) and logistic growth of populations recovering from three levels of exploitation (to the right of time $b$ ) and their relationships to model predictions of exponential growth. The shaded regions indicate where the logistic and exponential models give similar results.

levels of fishing mortality $(F)$. Note that this implies that the predicted rate of population change is zero in all cases. Removing the fishing mortality (time $b$ ) in each scenario reveals the potential rate of population increase $\left(r_{\text {achieved }}\right)$ that can result at the given population size. (This rate of change will be close to $r_{\text {conditional }}$ but will not be exactly equal to $r_{\text {conditional }}$ because the age structure will no longer be in equilibrium). The virgin population can realize no change in fishing mortality and continues at its carrying capacity in an equilibrium state $\left(r_{\text {achieved }}=r_{\text {conditional }}=r_{\text {predicted }}=0\right)$. The moderately fished population has been released from some level of fishing mortality, and the calculated value of $r_{\text {achieved }}$ represents the short-term growth potential of a population of that size when released from exploitation. In the depleted population, density-dependent compensation is at its maximum $\left(S_{0}\right.$ is at its maximum in our example), and once released from fishing pressure the population will recover at close to its maximal rate $\left(r_{\text {achieved }}\right.$ approaches $r_{\text {intrinsic }}$ and would equal $r_{\text {intrinsic }}$ if an equilibrium stable age structure were present). Thus, the instantaneous potential population growth rate $\left(r_{\text {achieved }}\right)$ depends on the size of the population. This is a property of all population models with density dependence. According to the logistic model being used in our example and assuming a stable age distribution, the population growth rate is related to the intrinsic rate of increase in the absence of fishing by 


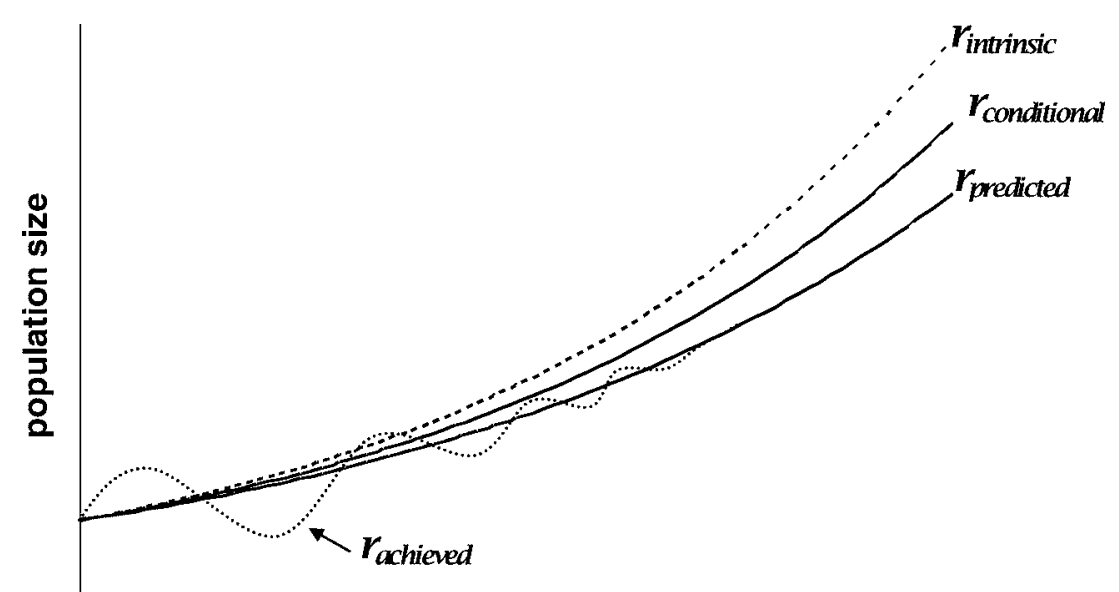

\section{time}

FIGURE 2.-Exponential population growth under fishing pressure $\left(r_{\text {predicted }}\right)$, under no fishing pressure $\left(r_{\text {conditional }}\right)$, and at the maximum rate $\left(r_{\text {intrinsic }}\right)$, all with a stable age distribution, and under fishing pressure without a stable age distribution $\left(r_{\text {achieved }}\right)$.

the linear relationship (Figure 3)

$$
r_{\text {conditional }}=r_{\text {intrinsic }} \cdot(1-N / K) \text {. }
$$

Note that $r_{\text {conditional }}$ is the per capita instantaneous rate of population growth; the instantaneous rate of population increase is

$$
\frac{d N}{d t}=r_{\text {conditional }} \cdot N=r_{\text {intrinsic }} \cdot(1-N / K) \cdot N,
$$

and the short-term population trajectory is

$$
N_{t+\Delta t}=N_{t} \cdot e^{r_{\text {intrinsic }}\left(1-N_{t} / K\right) \times \Delta t} .
$$

When fishing occurs on all ages at an instantaneous rate $F$ (per year),

$$
r_{\text {predicted }}=r_{\text {conditional }}-F=r_{\text {intrinsic }} \cdot(1-N / K)-F \text {. }
$$

Using the calculated $r_{\text {conditional }}$ in each population to project the population growth forward in time after the cessation of fishing along with equation (6) (and

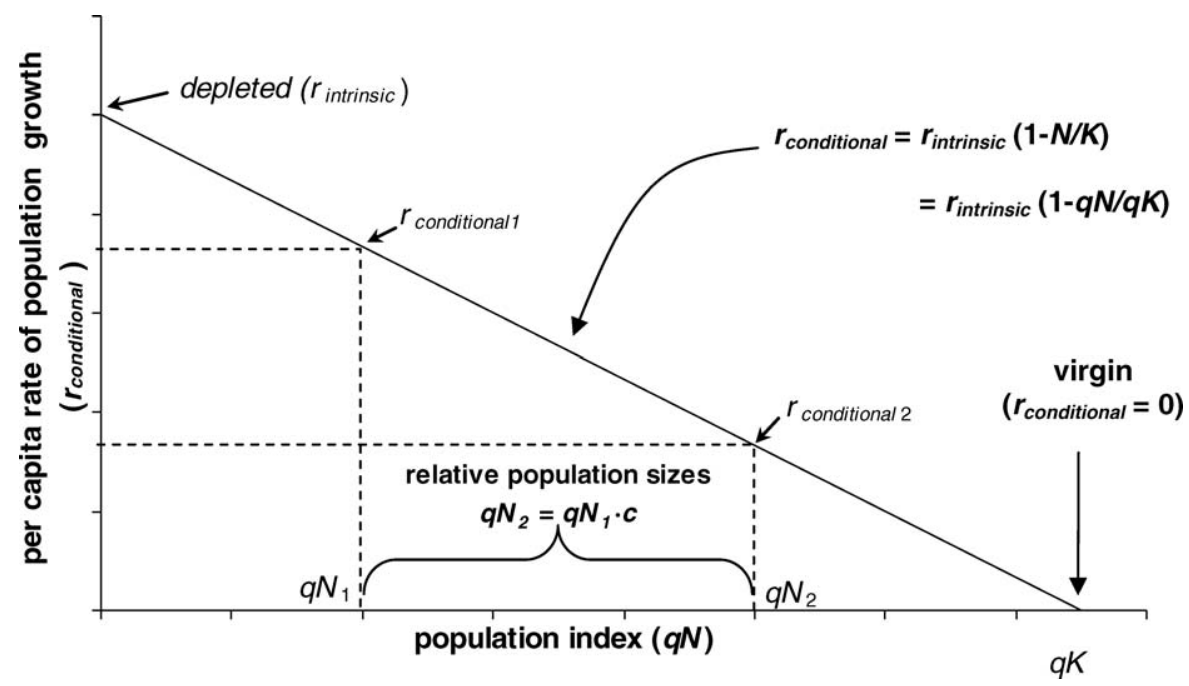

FIGURE 3.-The instantaneous per capita rate of population growth $\left(r_{\text {conditional }}\right)$ for a stock as a function of stock size under logistic growth when fishing mortality is zero. When fishing mortality occurs equally at all ages, $r_{\text {predicted }}=r_{\text {conditional }}-F=$ $r_{\text {intrinsic }} \cdot(1-N / K)-F$, where $N / K$ is the ratio of the population size to the virgin population size. The relationships used to derive equations (8)-(10) for $r_{\text {intrinsic }}$ based on survey indices are indicated; $q$ is a constant relating the index to actual population size. 
assuming a stable age distribution) further illustrates the density-independent nature of the Leslie matrix-life table model (Figure 1b). Exponential growth is predicted. This is realistic over the short term but becomes increasingly unrealistic as the stock grows larger. The value of $r_{\text {conditional }}$ is dependent on the life history parameters $\left(S_{0}\right.$ in our example), which, in turn, are dependent on stock size. The value of $r_{\text {conditional }}$ and the predicted exponential growth approximate the results from the more realistic logistic growth model only for a specific stock size and thus only for a short period of time. It is therefore only possible to compute $r_{\text {intrinsic }}$ from a demographic analysis if the model parameters represent conditions in a severely depleted population or if additional information is available. In all other cases, all that is known about the calculated rate of population increase $\left(r_{\text {conditional }}\right)$ is that it falls somewhere between 0 and $r_{\text {intrinsic }}$ (Figure 3).

\section{Uses of the Leslie Matrix}

The above is not meant to imply that a Leslie matrix has no value by itself. A demographic analysis can be used to check the validity of parameter estimates. For example, Grusha (2005) found that parameter values from the literature for a viviparous species, the cownose ray Rhinoptera bonasus, in Chesapeake Bay seemed reasonable but led to a life table prediction that the population would crash under no fishing pressure unless $S_{0}$ exceeded $100 \%$. In this case or that in which $S_{0}$ is required to be greater than adult survival, the life history parameters are suspect and need to be reevaluated. The situation is more complicated for an oviparous species, and we discuss this in Advances in Methodology.

Unfortunately, for most elasmobranchs, the information necessary to determine $r_{\text {intrinsic }}$ through classic demographic modeling is unavailable. Even in the most well-studied species, where age at maturity, fecundity, natural mortality, and even stock size are known, estimates of $S_{0}, F$, and the rate of population change $\left(r_{\text {achieved }}\right)$ are rare. These three parameters are closely linked. In situations where two of the three parameters are known, the Leslie model can be used to solve for the remaining one (assuming that all other model parameters are known). For example, in situations where $F$ can be assumed to be zero and $r_{\text {achieved }}$ is known, the model can be solved for $S_{0}$ (as in Vaughan and Saila 1976; Hoenig and Gruber 1990). Alternatively, if $F$ is assumed to be zero and $S_{0}$ is known, the model can be solved for the current rate of population change (in this case $r_{\text {predicted }}$ should equal $r_{\text {conditional }}$; we return to this case when we consider the lemon shark data in Applications to Elasmobranchs). If $F$ and
$S_{0}$ are not known, as in most cases, there are an infinite number of solutions to the model that will result in equilibrium (or a specified rate of population growth).

In situations where even less information is available, matrix models can be used to examine the effects of individual parameters while holding all the other parameters constant and accounting for initial conditions (age composition). This can provide insights into the dynamics of the population, such as how various age-specific harvesting strategies affect the dynamics (Cortés 1995; Heppell et al. 1999; Beerkircher et al. 2002), but it does not allow quantitative predictions if some parameters are unknown and fixed at arbitrary values.

\section{Pitfalls of Interpreting Demographic Analyses}

For many viviparous elasmobranchs, parameter estimates are available for the proportion mature at age, fecundity, longevity, and survival in the absence of fishing mortality for all but the youngest ageclass(es). In only a few cases are estimates of observed population growth available. For oviparous species, estimates of fecundity are harder to obtain and information on egg stage and first-year survival is scant.

Lacking direct information, many studies have used empirical relationships to obtain survival rates. These relationships are of two types: those that provide a single value (e.g., Pauly 1980; Hoenig 1983; Jensen 1996) and those that provide age-specific values (Peterson and Wroblewski 1984; Chen and Watanabe 1989) (Table 1). The assumptions behind the use of the two types of estimates are different and should result in different interpretations of model results.

Most studies use empirical relationships that provide a single survival rate that presumably pertains to most of the lifespan in the absence of fishing. When this value is used in a demographic analysis, it is assumed that first-year survival in a severely depleted population equals that of unfished adults and represents the maximum possible value, $S_{0, \max }$. The calculated rate of population increase is then often interpreted as $r_{\text {intrinsic }}$ on the assumption that all other parameters are known. Although this can be a useful upper-limit biological reference point, there is no guarantee that a stock can exhibit this degree of compensation and growth and thus no evidence that this represents $r_{\text {intrinsic }}$. The maximum achievable $S_{0}$ is likely to be species specific, lower than the survival rate for an adult, and a function of size at birth. Assuming that $S_{0}$ can reach adult levels will clearly tend to overestimate $r_{\text {intrinsic }}$, but to what degree is unknown. Thus, the rate of increase calculated by setting $S_{0}$ equal to the survival of unfished adults gives us an upper-bound proxy for 
TABLE 1.- Survival rates for barndoor skate as derived from methods commonly used in demographic analysis, with required parameters in parentheses. Estimates were made an age at maturity $\left(a_{\text {mat }}\right)$ of 6.5 years (Gedamke et al. 2004), von Bertalanffy growth parameters $\left(k, L_{\infty}\right.$, and $\left.t_{0}\right)$ of $0.14 /$ year, $166.3 \mathrm{~cm}$, and -1.29 year, respectively), an assumed maximum age $\left(a_{\max }\right)$ of 25 years, and a water temperature of $8.5^{\circ} \mathrm{C}$ (Myers et al. 1997). The methods used are as follows: Hoenig (1983), Pauly (1980), Jensen (1996), Chen and Watanabe (1989), and Petersen and Wroblewski (1984).

\begin{tabular}{rcccccc}
\hline Age & Hoenig $\left(a_{\max }\right)$ & $\left(L_{\infty}, k\right.$, water temperature) & Jensen $\left(a_{\text {mat }}\right)$ & Jensen $(k)$ & $\begin{array}{c}\text { Chen and Watanabe } \\
\left(\mathrm{age}, k, t_{0}\right)\end{array}$ & $\begin{array}{c}\text { Peterson and Wroblewski } \\
\text { (weight at age) }\end{array}$ \\
\hline 0 & 0.846 & 0.843 & 0.776 & 0.811 & 0.429 & 0.517 \\
1 & 0.846 & 0.843 & 0.776 & 0.811 & 0.600 & 0.649 \\
2 & 0.846 & 0.843 & 0.776 & 0.811 & 0.684 & 0.714 \\
3 & 0.846 & 0.843 & 0.776 & 0.811 & 0.733 & 0.752 \\
4 & 0.846 & 0.843 & 0.776 & 0.811 & 0.765 & 0.777 \\
5 & 0.846 & 0.843 & 0.776 & 0.811 & 0.787 & 0.808 \\
6 & 0.846 & 0.843 & 0.776 & 0.811 & 0.803 & 0.818 \\
7 & 0.846 & 0.843 & 0.776 & 0.811 & 0.844 & 0.826 \\
8 & 0.846 & 0.843 & 0.776 & 0.811 & 0.844 & 0.832 \\
9 & 0.846 & 0.843 & 0.776 & 0.811 & 0.844 & 0.837 \\
10 & 0.846 & 0.843 & 0.776 & 0.811 & 0.844 & 0.841 \\
11 & 0.846 & 0.843 & 0.776 & 0.811 & 0.844 & 0.844 \\
12 & 0.846 & 0.843 & 0.776 & 0.811 & 0.844 & 0.847 \\
13 & 0.846 & 0.843 & 0.776 & 0.811 & 0.844 & 0.844 \\
14 & 0.846 & 0.843 & 0.776 & 0.811 & 0.844 & 0.852 \\
15 & 0.846 & 0.843 & 0.776 & 0.811 & 0.844 & \\
\hline
\end{tabular}

$r_{\text {intrinsic }}$, that is, a quantity related to $r_{\text {intrinsic }}$ that may have some use for fisheries management.

In some studies, purported estimates of $r_{\text {intrinsic }}$ have been calculated by specifying age-specific survival rates, including first-year survival, utilizing the empirical relationships described by Chen and Watanabe (1989) or Peterson and Wroblewski (1984) (e.g., Simpfendorfer 2000; Beerkircher et al. 2002; Cortés 2002). However, these empirical relationships were not based on modeling severely depleted populations but rather describe "normal" or virginal survival. Therefore, analysis of a Leslie matrix based on these parameter values should result in a value of $r$ conditional of zero (i.e., the value corresponding to the average growth of a virgin population). Any departure from zero represents measurement error, not the intrinsic rate of population increase. The expectation in much of the literature is that estimates of population growth calculated from a demographic analysis with no fishing mortality will be positive and that the amount of fishing mortality that results in an equilibrium state $\left(r_{\text {predicted }}=0\right)$ represents the maximum sustainable fishing pressure that can occur before a species is at risk of extinction. This is false.

If we consider the hypothetical populations presented in Figure 1b, the error in this logic is clear. In the depleted population at time a, $r_{\text {conditional }}$ is equal to $r_{\text {intrinsic }}$, while in the virgin population $r_{\text {conditional }}$ is equal to zero. In our depleted scenario, the $F$ that results in equilibrium is the maximum sustainable fishing pressure. In contrast, the removal of a single fish per year (i.e., any $F>0$ ) in our virgin population will result in negative population growth under the Leslie (exponential) model, which does not allow for compensation. According to the widespread logic, this means that a virgin population is more susceptible to fishing pressure than a depleted population of the same species. This conclusion is obviously false and highlights the strong relationship between the results of a simple demographic model and the population size for which model parameters have been specified.

\section{Ranking Species}

A number of studies have suggested that demographic analyses can be used for comparative purposes, that is, to rank species according to their ability to withstand exploitation based on calculated values of the rate of population increase (Smith et al. 1998; Walker and Hislop 1998; Frisk et al. 2002; Gallucci et al. 2006). Therefore, it is worth examining whether a Leslie matrix or life table can be used to rank species if we cannot get unbiased estimates of the intrinsic rate of increase. We consider four proxies for $r_{\text {intrinsic }}$ found in the literature that differ in the way $S_{0}$ is computed. All methods are based on the assumption that all other parameters are known; $S_{0}$ is derived by

(1) setting it equal to a constant for all species (Frisk et al. 2002),

(2) setting it equal to the survival of unexploited adults,

(3) setting it equal to virgin survival as computed from the formulae of Chen and Watanabe (1989) or Peterson and Wroblewski (1984), or

(4) solving for it after setting adult survival equal to the square of the unexploited survival rate, that is, $\exp (-2 M)$ (Smith et al. 1998). 


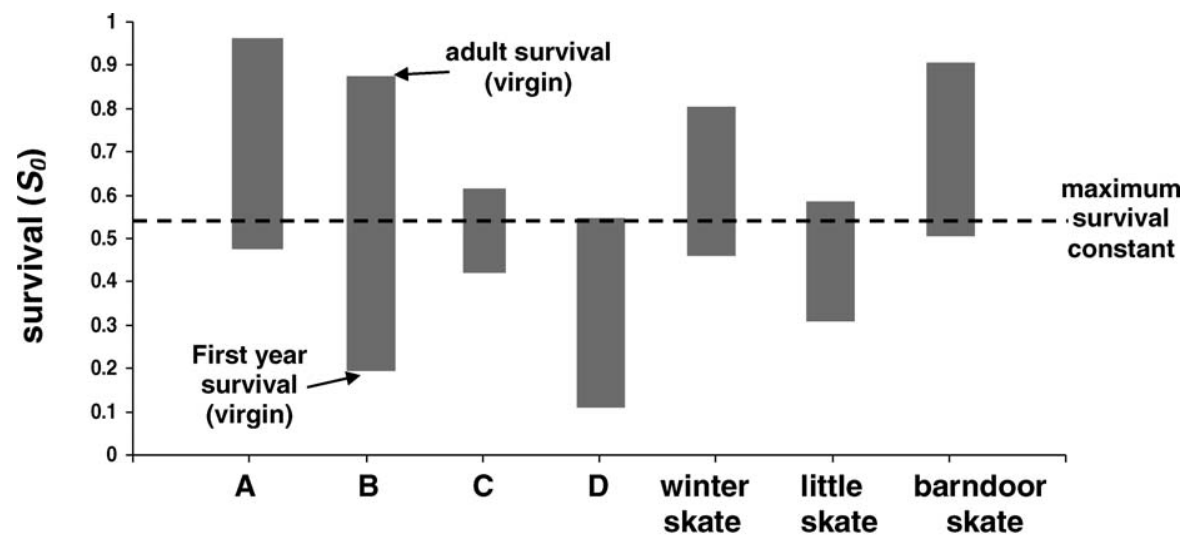

species

FIGURE 4.- Model for compensation in which all species attain the same maximum value of $S_{0}$ (indicated by the dashed line) regardless of first-year survival in a virgin population (below the dashed line) and adult survival in the absence of fishing (above the dashed line). Species A-D represent hypothetical populations. For the little skate, winter skate, and barndoor skate, adult survival is as in Frisk et al. (2002) and first-year survival was calculated according to the method of Peterson and Wroblewski (1984). Note the differing levels of compensation that are assumed when a constant $S_{0}$ is used.

Species vary in their size at birth and thus presumably in their first-year survival. However, under the first approach it is assumed that all of the populations being considered have the same maximum first-year survival (Figure 4). That is, although we know that the species vary in life history traits, this approach assumes they do not vary at all in the parameter that is likely to play the greatest role in determining a species' scope for compensation and therefore its intrinsic rate of increase. According to this model, differences among species in their intrinsic rate of increase are due to differences in fertility and adult survival only. We would argue that it is just as plausible (or more plausible) that the first-year survival of all species lies half way between virgin $S_{0}$ and virgin adult survival. In fact, the constant- $S_{0}$ approach has important implied assumptions regarding the scope of density-dependent compensation that are illustrated in Figure 4. Population A, the winter skate Leucoraja ocellata, and the barndoor skate are assumed to have almost no scope for compensation, while first-year survival in population D and the little skate L. erinacea can fully compensate in the sense of coming close to adult values. Species B resembles species D in having very low survival in virgin populations, but the firstyear survival of $D$ can rise to that of adults whereas the first-year survival of B can only reach half that of adult survival. It is not clear that this is a reasonable assumption. Therefore, this ranking of species' resiliency is conditional on the model of compensation being correct, which has not been demonstrated.
Many studies compute an upper-bound proxy for $r_{\text {intrinsic }}$ by setting first-year survival equal to virgin adult survival. This has the advantage of inducing a positive bias, so that upper bounds to the intrinsic rate of increase (and thus sustainable fishing mortality) can be obtained. That is, with this procedure we can conclude that certain levels of fishing mortality are not sustainable. However, this proxy may or may not be appropriate for ranking species' growth potential. Consider two shark species, the sand tiger Carcharias taurus and the tiger shark Galeocerdo cuvier. Both species have large maximum sizes and high longevities, but the former has two large pups while the latter has dozens of small pups. The sand tiger pups probably have high survival under virgin conditions, and it is not unreasonable to suppose that their survival could rise the small distance to adult survival. The tiger shark pups are much smaller and presumably have much lower survival under virgin conditions. It is less likely that their survival will approach the survival of adults. In other words, the potential scope of compensation may be markedly different even for these two apparently similar species. Thus, investigators can disagree over whether the use of this proxy for ranking species' population growth potential is reasonable.

Next, consider the estimates that result when firstyear survival is set equal to the Chen and Watanabe (1989) or the Peterson and Wroblewski (1984) estimates. All the estimates of population growth rate should be close to zero, so that all we are getting from 
this procedure is measurement error, which clearly is not a basis for ranking species.

Finally, consider the procedure of Smith et al. (1998). These investigators recognized the problems that result from the density-independent nature of demographic models and began the process of developing a methodology and a biological benchmark that would be comparable across species. The main assumption in their model is that a population being fished to produce maximum sustainable yield (MSY) will be at half the virgin stock size and have adult mortality of $Z=F+M=2 M$. They further assume that any density-dependent compensation occurs in $S_{\text {juv }}$ (the survival of juveniles) rather than in $S_{0}$. They then follow the methodology of Vaughan (1977) and solve the model for the value of $S_{\text {juv }}$ that results in equilibrium under the MSY fishing conditions. Finally, they remove the fishing mortality from the model and solve for the resulting instantaneous rate of population growth, which they term the "rebound potential" or $r_{2 M}$. In this way they attempt to standardize the level of density-dependent compensation in different species by deriving an estimate of $S_{\text {juv }}$ that is directly related to the life history parameters of the species. The validity of $r_{2 M}$ as a proxy for $r_{\text {intrinsic }}$ has not been established, but this approach has the potential to be used in comparative demography among species.

Although the Smith et al. (1998) approach has avoided some of the main pitfalls of demographic analysis by accounting for density-dependent compensation through clear logical assumptions, their procedure provides no indication of how far these rebound potentials are from $r_{\text {intrinsic }}$. Thus, managers are still faced with uncertainty about the maximum sustainable fishing pressure. To determine this, an actual estimate of $r_{\text {intrinsic }}$ is required.

\section{Advances in Methodology}

The absence of detailed information about a depleted population necessitates the use of alternative methods to estimate $r_{\text {intrinsic }}$. There are two aspects to the problem: (1) determining values of $r_{\text {conditional that }}$ correspond to a specific stock size and (2) determining $r_{\text {intrinsic }}$ from two or more values of $r_{\text {conditional }}$. The tools required to obtain an actual estimate of $r_{\text {intrinsic }}$ have long been in use in both fisheries and ecological research. Ricker (1975) summarized the early use of the logistic model for fishery assessment (and estimating $\left.r_{\text {intrinsic }}\right)$ when only limited data are available. Although he dealt with biomass models, the mathematics are the same if one substitutes numbers for biomass. We build our results on those presented by Ricker.

\section{Estimating $r_{\text {conditional }}$ for a Viviparous Species}

For viviparous species, we assume that the schedule of age-specific natural mortality for all ages above the first and age-specific fecundity are known for a particular population. Thus, everything is known except three parameters: first-year survival, fishing mortality, and the rate of population change, $r_{\text {predicted }}$ (or, more likely, $r_{\text {achieved }}$ ). As previously described, these three parameters are closely linked, and if two of the three are known the Leslie model or a life table can be used to solve for the third. For example, suppose the instantaneous natural mortality rate is estimated to be $0.2 /$ year and the instantaneous fishing mortality rate is determined from a tagging study to be $0.3 /$ year. Then all survivals except $S_{0}$ can be computed as $\exp (-0.2-$ $0.3)=0.6$. Suppose we know that the population is currently declining by $5 \%$ per year $\left(r_{\text {achieved }}=\right.$ $\log _{e}(0.95)=-0.051 /$ year) and we assume that $r_{\text {achieved }}$ is a reasonable estimate of $r$ predicted. Then the model can be solved for $S_{0}$ using Vaughan's (1977) method. We can analyze a Leslie matrix with the same fecundities and $S_{0}$ but with the other survivals adjusted to eliminate the fishing; in this case, survival for all ages except the first is $\exp (-0.2)=0.8$. This provides an estimate of $r_{\text {conditional }}$. When fishing mortality applies to all ages, there is a simpler solution:

$$
r_{\text {predicted }}=r_{\text {conditional }}-F \text {. }
$$

In our example, fishing mortality applies to all ages except the first (i.e., the young of the year) and thus equation (8) is a reasonable approximation. Hence, $r_{\text {conditional }}$ for our example is $-0.051+0.30=0.249$ / year.

\section{Estimating $r_{\text {conditional }}$ for an Oviparous Species}

In oviparous species with an egg stage lasting 1 year, the additional life stage adds a level of complexity to the demographic model. For these species an additional row and an additional column must be added to the matrix and another parameter, egg stage survival $\left(S_{\text {egg }}\right)$, must be included in the model. In most cases there is considerable uncertainty in the estimates of both $S_{\text {egg }}$ and fecundity. However, assuming that the proportion mature at age is known, all that is needed is the product of fecundity, $S_{\text {egg }}$, and $S_{0}$ rather than the value of each individual parameter. That is, doubling one parameter (e.g., $S_{0}$ ) and halving another (fecundity or $S_{\text {egg }}$ ) results in the same rate of population growth. This has an important implication for the reasonableness of parameter values. An $S_{0}$ estimate of, say, $200 \%$, does not imply a poor fit of the model to the data. Rather, it implies that the apportionment of recruitment (fecundity $\cdot S_{0} \cdot S_{\text {egg }}$ ) to individual parameters is in error, and 
this is inconsequential for projecting short-term changes in the population. On the other hand, if one constrains the values of $S_{\text {egg }}$ and $S_{0}$ to be no more than 1 , one can determine the minimum value for fecundity required for the population to grow at the observed rate.

The overall analysis for an oviparous species is virtually identical to that for a viviparous species, but instead of solving the model for $S_{0}$ as in the Vaughan (1977) method one solves it for the product of $S_{\text {egg }}, S_{0}$, and fecundity. Fishing pressure is then removed from the model as in the example for viviparous species to generate an estimate of $r_{\text {conditional }}$. We illustrate this approach in our barndoor skate example (see Application to Elasmobranchs).

\section{Estimating $r_{\text {intrinsic }}$}

The second step is to use the estimates of $r$ conditional to estimate $r_{\text {intrinsic }}$. Here we make use of the linear relationship between $r_{\text {conditional }}$ and population size under the logistic model (equation 4) and utilize observations on population behavior at two or more stock sizes. There are two cases to consider. First, suppose that an estimate of the conditional rate of population growth $\left(r_{\text {conditional }}\right)$ is available, along with an estimate of the corresponding population size $(N)$ as a fraction of the virgin population size $(K)$. Then, by virtue of the fact that the conditional rate is a linear function of population size (Figure 3; equation 4) and the value of $r$ conditional is zero when $N=K$, we can solve for the intercept as

$$
r_{\text {intrinsic }}=\frac{r_{\text {conditional }}}{1-\frac{N}{K}}=\frac{K r_{\text {conditional }}}{K-N} .
$$

This methodology differs from all the current approaches to demographic analysis of elasmobranchs in that the results do not simply provide a snapshot of population growth under a given set of circumstances but rather define the overall relationship between population size and the per capita rate of population growth according to the theory of logistic growth.

In the second case, we have information from observation of the population at two or more stock sizes but do not know how those stock sizes relate to the virgin population level. Instead, we know the relative size of the population at the various times. Assume we have survey data that give relative population size ( $q N$, where $q$ is the catchability coefficient [a constant relating the index to the population size $N]$ ) at two points in time along with the corresponding information necessary to calculate $r_{\text {conditional }}$ (Figure 3). Since we are assuming that the relationship between $r$ conditional and $N$ is linear and now know the ratio of the $q N \mathrm{~s}$, we can derive the following equations:

$$
r_{\text {conditional }_{1}}=\frac{r_{\text {intrinsic }}\left(q K-q N_{1}\right)}{q K}
$$

and

$$
r_{\text {conditional }_{2}}=\frac{r_{\text {intrinsic }}\left(q K-c q N_{1}\right)}{q K},
$$

where $c$ is a constant known from the two surveys $\left(N_{2}=c N_{1}\right)$. Although these two equations have three unknowns $\left(K, r_{\text {intrinsic }}\right.$, and $\left.N_{1}\right)$, a unique solution for $r_{\text {intrinsic }}$ results, namely,

$$
r_{\text {intrinsic }}=\frac{\left(r_{\text {conditional }_{2}}-c r_{\text {conditional }_{1}}\right)}{1-c} .
$$

Therefore, to obtain an estimate of $r_{\text {intrinsic }}$ we can calculate $r_{\text {conditional }}$ at two points in time for which the relative population sizes are known.

Note that if enough values of $r_{\text {conditional }}$ with the corresponding (relative) population sizes are known, one can derive an empirical relationship between the population growth rate and size and extrapolate back to zero population size. This avoids having to make the assumption of a linear relationship. Alternatively, if an extensive time series of indices of abundance is available along with the corresponding (schedules of) fishing mortality, it may be possible to fit a model that incorporates density dependence directly. This may be more efficient than the two-stage procedure described here. Although such data will rarely be available, exploration of these ideas is exciting (but beyond the scope of this paper).

\section{Application to Elasmobranchs}

To illustrate the points made in this paper, we consider two species of elasmobranch: the lemon shark and the barndoor skate. For both species sufficient data are available to illustrate the methods presented and generate at least preliminary estimates of $r_{\text {intrinsic }}$. Our intent here is to demonstrate the methodology; a more detailed analysis is being conducted for both species.

\section{Lemon Shark}

The lemon shark is one of only a few elasmobranch species for which empirical estimates of $S_{0}$ are available. Estimates of $S_{0}$ were obtained for differing stock sizes between 1995 and 1999 through a tagging and depletion study in Bimini, Bahamas (Gruber et al. 2001; S. H. Gruber, unpublished). The results from this study suggest that density dependence is occurring in the lemon shark nursery area, with an apparently inverse relationship between first-year survival and the 


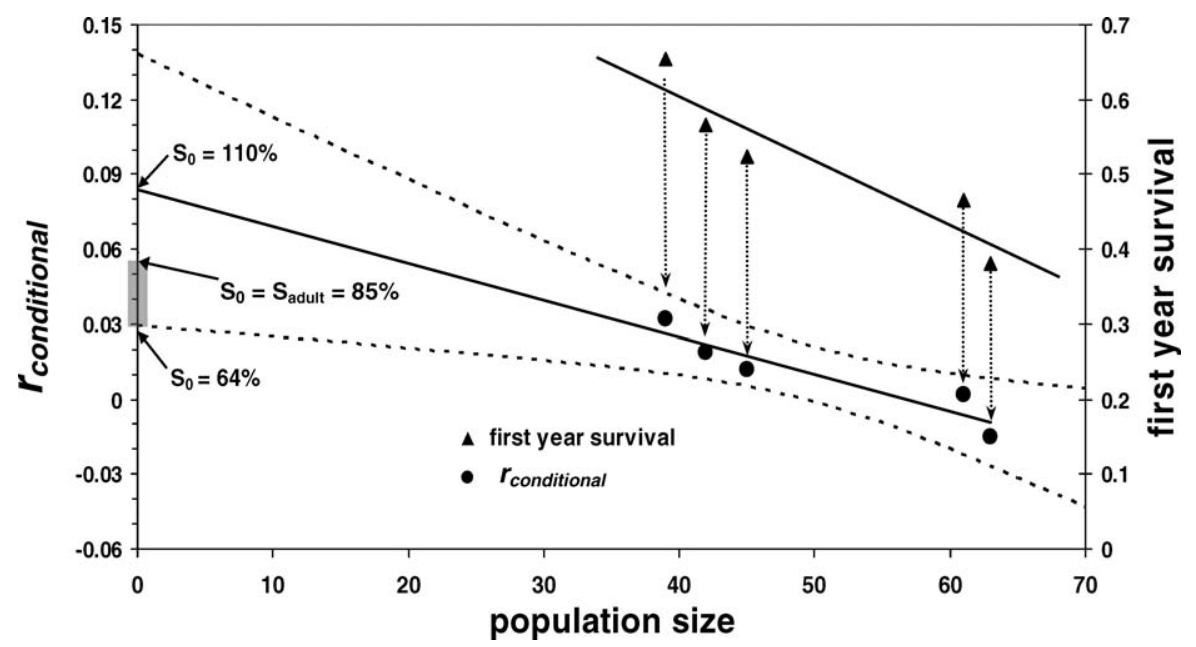

FIGURE 5.- The upper solid line shows the relationship between the first-year survival $\left(S_{0}\right)$ of lemon sharks and the population size of juveniles at Bimini as determined from a tagging depletion study. Note that one additional data point has been calculated beyond those presented by Gruber et al. (2001). The lower solid line shows the relationship between $r_{\text {conditional }}$ and the corresponding population size (the first-year survival used to calculate each value of $r_{\text {conditional }}$ is also indicated). The $y$-intercept represents the estimated value of $r_{\text {intrinsic }}$, and the dotted lines represent the $95 \%$ confidence intervals for the regression. Also shown on the $y$-axis are the first-year survival rates corresponding to three values of $r_{\text {conditional }}$ The shaded area shows the range of possible values for $r_{\text {intrinsic }}$ that is consistent with the data and with the maximum value of $S_{0}$ not exceeding adult survival.

size of the age-0 population (Figure 5). Since there is no directed fishery for lemon shark juveniles at Bimini, we assume that for juveniles $F$ is equal to zero. A prebreeding, birth-pulse, female-only Leslie matrix model was constructed with an age at maturity of 12 years and a longevity of 25 years. Lemon sharks are believed to have biennial parturition with an average of 12 pups, resulting in a fecundity term for the matrix of 3 females per year (Feldheim et al. 2002). Since lemon sharks have been shown to stay in the nursery lagoon at Bimini for the first few years of life (Morrissey and Gruber 1993), second-year survival was set at the mean of first-year and adult survival.

The model was then solved for the population growth $\left(r_{\text {conditional }}\right)$ that would occur given each estimate of first-year survival (note that this implies setting survival after the second year equal to that which would occur in the absence of fishing; in this case we used $S=0.85$ based on the Hoenig (1983) maximum-age formulation). The relationship between each year's estimated population size and $r_{\text {conditional }}$ allows us to extrapolate to a population growth rate as the population size approaches zero (i.e., $r_{\text {intrinsic }}$ ) of $0.08 /$ year (Figure 5). As a check on the feasibility of this estimate, the model was solved for the $S_{0}$ that would be required for the population to grow at a rate of $0.08 /$ year assuming $F=0$. Given the life history parameters used in our model, $S_{0}$ would have to be $110 \%$ for the population to grow at our estimated value of $r_{\text {intrinsic }}$. Clearly, this is infeasible and not surprising given an extrapolation from five data points. However, if we use the lower confidence limit from our relationship between $r_{\text {conditional }}$ and population size, the estimate of $r_{\text {intrinsic }}$ is $0.03 /$ year, which would require a first-year survival of $64 \%$ (Figure 5). Assuming that $S_{0}$ cannot be greater than adult survival (85\%), we now have an upper bound for $r_{\text {intrinsic }}$ of $0.06 /$ year and therefore an overall potential range of $r_{\text {intrinsic }}$ for the lemon shark of 0.03-0.06/year.

\section{Barndoor Skate}

The barndoor skate was reported to be potentially on the brink of extinction in 1998 (Casey and Myers 1998). As international fishing fleets were heavily fishing the Northwest Atlantic, National Marine Fisheries Service (NMFS) survey indices for the barndoor skate in the Gulf of Maine and southern New England went from highs of nearly 0.8 fish/tow in the early 1960s to lows of nearly zero for the 1970s and 1980s. In 1994, three large areas on Georges Bank (off Massachusetts) were closed to all mobile fishing gear. Since then, survey indices for the barndoor skate have been increasing at an annual rate of approximately $43 \%$, providing an observed rate of growth of $\log _{e}(1.43)=0.36 /$ year, which we assume is a reasonable estimate of $r_{\text {achieved }}$. An estimate of $F=$ $0.05 /$ year was generated by using a modification of the nonequilibrium form of the Beverton-Holt mean length 


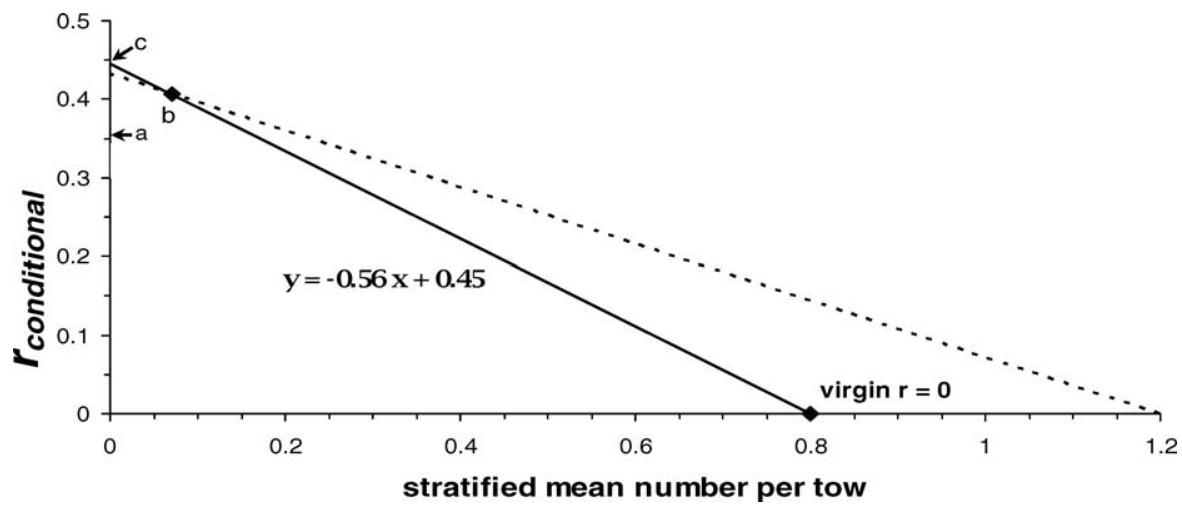

FIGURE 6.-Relationship between population growth rate and the NMFS survey index of population size for the barndoor skate (solid line). Point a indicates the value of $r_{\text {achieved }}$ based on the change in the survey index over time, point $\mathrm{b}$ indicates the calculated value of $r_{\text {conditional }}$ at an abundance of $0.07 \mathrm{fish} / \mathrm{tow}$, and point c indicates the estimated value of $r_{\text {intrinsic }}$. The dashed line indicates the slight reduction in $r_{\text {intrinsic }}$ that would occur if the virgin population size ( 0.8 fish/tow) is an underestimate.

mortality estimator (Gedamke and Hoenig 2006), which incorporates recruitment variability (Gedamke 2006) to estimate a total instantaneous mortality rate (Z) of 0.23/year. Subtracting the Pauly (1980) estimate for instantaneous natural mortality $(M)$ of 0.18 /year results in an estimate for $F$ of $0.05 /$ year. We assume that this fishing mortality pertains to all ages because barndoor skates are born at a large size of nearly $20 \mathrm{~cm}$ total length. Given an $F$ of $0.05 /$ year, we solved the Leslie matrix model for the product of fecundity, $S_{\text {egg }}$, and $S_{0}$ that would be necessary for the population to be growing at the observed rate of 0.36/year. Assuming that $S_{0}$ and $S_{\text {egg }}$ are no more than 1, a minimum value for fecundity of 23 female eggs per year would be required for the population to grow at the observed rate. This is consistent with the fecundity estimates for similar oviparous species (Musick and Ellis 2005). Fishing pressure was then removed from the model to generate an estimate of $r_{\text {conditional }}$ of 0.41 year (equation 8).

Although the barndoor skate population is clearly depleted and our estimated $r_{\text {conditional }}$ should approximate $r_{\text {intrinsic }}$, we can take our analysis one step further by considering the relative stock sizes from the NMFS survey data. In 1994 the index was at approximately $10 \%$ of the high recorded at the start of the time series in 1963. If we treat the 0.8 -fish/tow maximum observed in 1963 as representing the virgin condition (i.e., $r_{\text {conditional }}=0$ ) and plot it together with our estimate of $r_{\text {conditional }}$ at the corresponding survey index ( 0.07 fish/tow), we get two points that uniquely define a straight line. Extrapolating to a stock size of zero gives an estimate of $r_{\text {intrinsic }}$ of $0.45 /$ year (Figure 6). If the index in 1963 represents the situation with some fishing, then the rightmost point in Figure 6 should be farther to the right, thus lowering the $y$-intercept. Because the barndoor skate population was so low in 1994, the uncertainty in the virgin stock size affects the estimated value of $r_{\text {intrinsic }}$ only slightly.

The increase in the barndoor skate population size observed in the NMFS surveys provides compelling evidence that the skate population can grow rapidly, in excess of $40 \%$ per year. The corrections for fishing mortality and population size may be less compelling at this point but clearly demonstrate that such calculations are feasible.

\section{Discussion}

The use of a Leslie matrix or life table demographic analysis for an elasmobranch population is generally challenging because of limited information on population trends, fishing mortality, and life history parameters. The simplest case is the one in which the intrinsic rate of increase can be estimated from observations on a severely depleted population released from fishing pressure. This was approximately the case for the barndoor skate. But, in general, estimation of the intrinsic rate of increase is an involved process that requires some information on relative stock size. Most reported estimates of the intrinsic rate of population increase for elasmobranchs are ill founded because the analysis does not take population size into consideration.

Studies can take one of three approaches when direct observation of maximum population growth is not possible. The first is to determine the life history parameters, rate of population change, and fishing mortality that occur in an extremely depleted population. In this case, the model can be used to estimate the rate of population growth that would occur if the 
population were released from fishing pressure. For most species, however, not enough information is available and one of two alternative approaches must be taken. A basic demographic analysis only provides an instantaneous rate of population growth (not $r_{\text {intrinsic' }}$, except in special circumstances) for a specific set of life history parameters associated with a specific fishing mortality and population size. As we show in this paper, with additional data it is possible to estimate $r_{\text {intrinsic }}$ from the results of basic demographic analyses by extrapolating the conditional population growth rate $\left(r_{\text {conditional }}\right)$ to zero population size. When the data are insufficient for estimating $r_{\text {intrinsic }}$, however, the only recourse is to be content with calculating a proxy for $r_{\text {intrinsic }}$ (such as an upper bound) and present it as such.

We believe that the basic requirement for estimating $r_{\text {intrinsic }}$ from a Leslie matrix (i.e., modeling a depleted population) is largely being overlooked and that there are three different aspects to the problem. First, there is understandable confusion because the standard texts do not emphasize the relationships between $r_{\text {intrinsic' }}$,

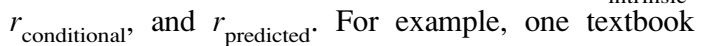
(Gotelli 1998) defines the symbol $r$ as the intrinsic rate of increase and then estimates $r$ for a virgin population and after $50 \%$ of the population is removed. We see these problems translated to the elasmobranch literature, with such findings as positive values for the "virginal intrinsic rate of increase" (implying that an unexploited population will increase exponentially forever; Xiao and Walker 2000).

Second, the perception that an intrinsic rate of increase can easily be obtained from a Leslie matrix or life table negates the importance of advancing the field through development of new techniques. The importance of collecting additional information is thus missed. Third, the results from demographic analyses are being used as the basis for management recommendations that will not be effective in achieving their stated goals. A demographic model for any species that uses "normal" age-specific survival rates (e.g., those based on Chen and Watanabe 1989 or Peterson and Wroblewski 1984) should result in a population growth rate of zero and not an estimate of $r_{\text {intrinsic }}$. Any other calculated value results from measurement error or noise in the data and is thus probably a substantial underestimate. The resulting conclusion that only extremely low fishing mortality can be tolerated and that modest levels of bycatch may lead to stock collapse and possibly extinction is simply untenable because the analysis is logically flawed. One may argue that this procedure is "conservative" in the short term, but it is not a rational basis for management. Scientifically, the results are erroneous and their use may lead to misunderstanding and to scientists' loss of credibility.

\section{Acknowledgments}

We thank Kyle Shertzer, Douglas Vaughan, Ken Goldman, Enric Cortés, and one anonymous reviewer for helpful comments. Financial support from the National Marine Fisheries Service Southeast Fishery Science Center is gratefully acknowledged. This is VIMS contribution number 2822.

\section{References}

Beerkircher, L., M. Shivji, and E. Cortés. 2002. A Monte Carlo demographic analysis of the silky shark (Carcharhinus falciformis): implications of gear selectivity. U. S. National Marine Fisheries Service Fishery Bulletin 101:168-174.

Casey, J. M., and R. A. Myers. 1998. Near extinction of a large, widely distributed fish. Science 281:690-692.

Caswell, H. 2001. Matrix population models, 2nd edition. Sinauer Associates, Sunderland, Massachusetts.

Chen, S., and S. Watanabe. 1989. Age dependence of natural mortality coefficient in fish population dynamics. Bulletin of the Japanese Society of Scientific Fisheries 55:205-208.

Cortés, E. 1995. Demographic analysis of the Atlantic sharpnose shark, Rhizoprionodon terraenovae, in the Gulf of Mexico. U. S. National Marine Fisheries Service Fishery Bulletin 93:57-66.

Cortés, E. 2002. Incorporating uncertainty into demographic modeling: application to shark populations and their conservation. Conservation Biology 16:1048-1062.

Cortés, E. 2004. Life history patterns, demography, and population dynamics. Pages 449-469 in J. C. Carrier, J. A. Musick, and M. R. Heithaus, editors. Biology of sharks and their relatives. CRC Press Boca Raton, Florida.

Feldheim, K. A., S. H. Gruber, and M. V. Ashley. 2002. Breeding biology of lemon sharks at a tropical nursery lagoon. Proceedings of the Royal Society of London B 269:1655-1662.

Frisk, M. G., T. J. Miller, and M. J. Fogarty. 2002. The population dynamics of little skate Leucoraja erinacea, winter skate Leucoraja ocellata, and barndoor skate Dipturus laevis: predicting exploitation limits using matrix analyses. ICES Journal of Marine Science 59:576-586.

Gallucci, V. F., I. G. Taylor, and K. Erzini. 2006. Conservation and management of exploited shark populations based on reproductive value. Canadian Journal of Fisheries and Aquatic Sciences 63:931-942.

Gedamke, T. 2006. Developing a stock assessment for the barndoor skate Dipturus laevis in the Northeast United States. Doctoral dissertation. College of William and Mary, Gloucester Point, Virginia.

Gedamke, T., W. D. DuPaul, and J. A. Musick. 2004. Observations on the life history of the barndoor skate, Dipturus laevis, on Georges Bank (western North Atlantic). Journal of Northwest Atlantic Fishery Science 35:67-78. 
Gedamke, T., and J. M. Hoenig. 2006. Estimating mortality from mean length data in nonequilibrium situations, with application to the assessment of goosefish. Transactions of the American Fisheries Society 135:476-487.

Gotelli, N. J. 1998. A primer of ecology, 2nd edition. Sinauer Associates, Sunderland, Massachusetts.

Gruber, S. H., J. R. C. de Marignac, and J. M. Hoenig. 2001. Survival of juvenile lemon sharks at Bimini, Bahamas, estimated by mark-depletion experiments. Transactions of the American Fishery Society 130:376-384.

Grusha, D. S. 2005. Investigation of the life history of the cownose ray, Rhinoptera bonasus (Mitchill 1815). Master's thesis. College of William and Mary, Gloucester Point, Virginia.

Heppell, S. S., L. B. Crowder, and T. R. Menzel. 1999. Life table analysis of long-lived marine species with implications for conservation and management. Pages 137-148 in J. A. Musick, editor. Life in the slow lane: ecology and conservation of long-lived marine animals. American Fisheries Society, Symposium 23, Bethesda, Maryland.

Hoenig, J. M. 1983. Empirical use of longevity data to estimate mortality rates. U. S. National Marine Fisheries Service Fishery Bulletin 82:898-903.

Hoenig, J. M., and S. H. Gruber. 1990. Life history patterns in the elasmobranchs: implications for fisheries management. Pages 1-16 in H. L. Pratt, Jr., S. H. Gruber, and T. Taniuchi, editors. Elasmobranchs as living resources: advances in the biology, ecology, systematics, and the status of fisheries. NOAA Technical Report NMFS 90.

Jensen, A. L. 1996. Beverton and Holt life history invariants result from optimal trade-off of reproduction and survival. Canadian Journal of Fisheries and Aquatic Sciences 53:820-822.

Mollet, H. F., and G. M. Cailliet. 2002. Comparative population demography of elasmobranchs using life history tables, Leslie matrices, and stage-based matrix models. Marine and Freshwater Research 53:503-516.

Morrissey, J. F., and S. H. Gruber. 1993. Home range of juvenile lemon sharks, Negaprion brevirostri. Copeia 1993:425-434.

Musick, J. A., and J. K. Ellis. 2005. Reproductive evolution of Chondrichthyes. Pages 45-79 in W. C. Hamlett, editor. Reproductive biology and phylogeny of Chondrichthyes: sharks, batoids, and chimaeras. Science Publishers, Plymouth, UK.
Pauly, D. 1980. On the interrelationships between natural mortality, growth parameters, and mean environmental temperature in 175 fish stocks. Journal du Conseil International pour l'Exploration de la Mer 39:175-192.

Peterson, I., and J. S. Wroblewski. 1984. Mortality rate of fishes in the pelagic ecosystem. Canadian Journal of Fisheries and Aquatic Sciences 41:1117-1120.

Ricker, W. E. 1975. Computation and interpretation of biological statistics of fish populations. Fisheries Research Board of Canada Bulletin 191.

Sibley, R. M., D. Barker, M. C. Denham, J. Hone, and M. Pagel. 2005. On the regulation of populations of mammals, birds, fish, and insects. Science 309:607-610.

Simpfendorfer, C. A. 2000. Predicting population recovery rates for endangered western Atlantic sawfishes using demographic analysis. Environmental Biology of Fishes 58:371-377.

Sminkey, T. R., and J. A. Musick. 1996. Demographic analysis of the sandbar shark, Carcharhinus plumbeus, in the western North Atlantic. U. S. National Marine Fisheries Service Fishery Bulletin 94:341-347.

Smith, S. E., D. W. Au, and C. Show. 1998. Intrinsic rebound potentials of 26 species of Pacific sharks. Marine and Freshwater Research 49:663-678.

Vaughan, D. S. 1977. Confidence intervals on mortality rates based on the Leslie matrix. Pages 128-150 in W. Van Winkle, editor. Assessing the effects of power-plantinduced mortality on fish populations. Pergamon New York.

Vaughan, D. S., and S. B. Saila. 1976. A method for determining mortality rates using the Leslie matrix. Transactions of the American Fishery Society 105:380383.

Walker, P. A., and J. R. G. Hislop. 1998. Sensitive skates or resilient rays? Spatial and temporal shifts in ray species composition in the central and northwestern North Sea between 1930 and the present day. ICES Journal of Marine Science 55:392-402.

Xiao, Y., and T. I. Walker. 2000. Demographic analysis of gummy shark (Mustelus anarcticus) and school shark (Galeorhinus galeus) off southern Australia by applying a generalized Lotka equation and its dual equation. Canadian Journal of Fisheries and Aquatic Sciences 57:214-222. 